\title{
Research on Food Safety RFID Anti-collision Algorithm
}

\author{
Niu Xiang-jie \\ School of Computer and Information Engineering \\ Beijing University of Agriculture \\ Beijing, 102206, China
}

\author{
Li Hua \\ School of Economics\&Management, \\ Beijing University of Agriculture \\ Beijing, 102206, China(Corresponding author)
}

\begin{abstract}
As an effective way to control the quality and safety of agricultural products, traceability system is aroused growing world attention. On the purpose of retrospective quality, this paper constructs a food safety management and quality traceability system from IT view. The paper makes a detail analysis of implementation process of RFID Anticollision algorithm. This paper produces details about the anticollision mechanism in tags identification and proposes a dynamic frame time slot anti-collision algorithm, which fulfils the function of tags identification in system food safety quality trace.
\end{abstract}

Keywords- traceability system; quality and safety of food; RFID Anti-collision

\section{INTRODUCTION}

In the recent years, the food safety crisis-such as livestock and poultry diseases, serious agricultural residual drugs and the increasing imports of foreign food materials occurs frequently, which caused widespread concern in the world[1 2]. How to effectively manage the production of livestock and poultry products is becoming an extremely urgent global issue[3 4]. The mobile RFID technology is the emerging technology of RFID technical field, which through mobile devices and communication networks to realize the information exchange with RFID tag object. Different from common RFID technology, the mobile RFID does not require wires to fix Write-reader, and the Write-reader installed on the mobile device, mobile phone or PDA in order to reduce the number of required Write-readers in certain coverage. Write-readers are connected to the wireless network or the Internet through mobile devices, and read the back-end database storage items labeled information[5]. Currently, Organizations and associations such as NFC, ETSI and EPCglobal are starting the researches on mobile RFID. In addition, the technology combining RFID with IPv6 and LWAN network technology is the new direction of the future development of RFID technology. This paper mainly focuses on the tags collision issues in food safety surveillance system and proposes an anti-collision algorithm to ensure the application of food safety RF technology.

\section{RESEARCH ON FOOD SAFETY RFID ANTI-COLLISION} ALGORITHM

\section{A. The reasons of the collision in RFID identification}

In the writing and reading of food safety RFID the communication between the writer-reader and tags is wireless. In primary RFID system the tags can only be identified one by one and most of them are contact. When there are several tags in the area of one writer-reader, if there is no multiple channels access control mechanism, the multiple tags may want to send signals to the writer-reader simultaneously and the collision of the signals will happen. The writer-reader can't work normally, as shown in figure 1.

In the existing food safety application of the RFID system, the effective anti-collision algorithm is necessary especially in large scale food safety application the writerreader usually needs to identify multiple tags at the same time. The effective anti-collision technology in communication will reduce collision and misreading rate and leakage reading rate to improve the system performance. The existing anti-collision technique usually applies software to implement which is anti-collision algorithm. The software can reduce cost and easily be implemented.

There are four common multi-channels access methods: Space Division Multiple Access (SDMA), Frequency Division Multiple Access (FDMA), Code Division Multiple Access (CDMA) and Time Division Multiple Access (TDMA).

The Space Division Multiple Access technique uses the channels partitioned by space which is a channel compatibilization method can achieve the multiplex of frequencies. The method can reduce the resource cost by the writer-reader as less as possible and dispatch the residual resource to other writer-reader to form antenna coverage area. Thus the channels forming by a serial of writer-reader will increase and be multiplex. The disadvantage of SDMA is the cost which restricts its application.

The Frequency Division Multiple Access technique distributes multiple signals in the same time slot to different frequency channel and different signals have different carrier frequencies. Then the filter is used to choose useful signal and suppress the reference signal which makes each channel can be used at the same time. In RFID system, the multiplex is implemented by a harmonic emission frequency converter. Each tag only responds to one signal in multiple carrier frequencies.

The Code Division Multiple Access technique is based on pseudo random codes which apply the data in the whole spectrum with spread spectrum modulation technology. The CDMA is ideal signal anti-collision method in many communication manners. However in RFID system it is too complex and needs too many computations for tags.

The Time Division Multiple Access technique uses crossbit pulses in different channels or time slots to transmit several signals in the communication media which is widely applied in digital signal system. This technique is also 
utilized in RFID system. The anti-collision algorithm based on Aloha and binary tree is derived from TDMA. The main research in this paper is on the basic of Aloha.

\section{B. Tag identification anti-collision algorithm based on dynamic Aloha}

(1) Pure Aloha anti-collision algorithm

The writer-reader sends a request command which will be responded by nearby tags. All of the tags will send IDs to the writer-reader. At this more than two tags will send the IDs simultaneously and collision occurs. If there is only one tag sent, the tag will be detected as shown in figure 2 . The algorithm will cause 'tag hungry'. When the quantity of the tags is large the collision will continually happen and no tags will be detected. The performance of the algorithm will drop dramatically with the increasing amount of tags. The utilization of the channel is only $18.4 \%$.

(1) Frame time slot Aloha

In frame time slot Aloha algorithm the time is partitioned into discrete time intervals, that is time slot. A frame includes fixed number of time slot. When the writer-reader sends request to write or read, the request contains the size of the frame and some random numbers. The tag will choose the frame to send ID according to the rand number in order to reduce the collision range and increase the channel utilization. The operation of the algorithm is as shown in table 1.

The table 1 is the identification process in frame time slot Aloha. The size of the frame is fixed 4 time slots in this process. In the first frame, T1 and T3 will send ID in the first time slot. T2 and T5 will send ID in the third time slot. T4 will send ID in the fourth time slot and the second time slot is ideal due to no tags send data. In this frame only T4 can be correctly identified in the fourth time slot. There are two tags sending ID simultaneously in the first and third time slot, so the collision will happen. In the second frame, T3 and T2 will be identified in the first and third time slot respectively. The collision will happen in the second time slot due to T1 and T5. The fourth time slot is idle. So on and so forth until all of the tags are identified.

(2) Dynamic frame time slot Aloha in the paper

In the dynamic frame time slot Aloha algorithm, the size of the frame is determined by the previous frame depending on the successful writing-reading time slot, collision time slot and idle time slot. In the operation of the algorithm, each frame will set an upper threshold and a lower threshold. If the collision rate exceeds the upper threshold the writerreader will increase the time slot. If the collision rate is less than the lower threshold, the writer-reader will reduce the time slot. The dynamic adjustment will cause the waste of time slot but it will reduce parts of the collision and improve the performance. The detailed identification process is as shown in table 2.

In the identification process shown in table 2, there are 7 tags in the area of the writer-reader. There are 3 time slots in the first frame. T1 and T3 will send ID in the first time slot. T2 and T5 will send ID in the second time slot and T4, T5 and T7 will send ID in the third time slot. The writer-reader will detect the collisions in the entire time slot and the collision rate can be up to $100 \%$. Thus in the identification of second frame, it will increase 3 time slots. The dynamic adjustment will decrease the collision rate and increase the identification efficiency.

\section{ANALYSIS OF THE EXPERIMENT RESULTS}

Following is the comparison between the traditional algorithm and the algorithm proposed in this paper by simulation. The performance of RFID tags anti-collision algorithm is evaluated by the collision time slots, idle time slots and readable time slots. The readable time slots in this paper are the same as those in the traditional algorithm, so they are not compared. The below simulations are about the collision time slots and the idle time slots.

(1) Performance comparison when the number of the tags is the same

The simulation in figure 3 describes the operation results of the proposed algorithm and the traditional algorithm when the number of the tags is different. Figure 5-1(a) is the comparison of collision time slot which shows the proposed algorithm is better. The idle time slots are far less than that in traditional algorithm from figure 3(b). In figure 3(c) the collision time slots and idle time slots are more then the proposed algorithm. Thus generally proposed algorithm is better than traditional algorithm.

1) The effect of the departure rate on the identification performance

When the arrival rate $\mathrm{RA}=0.5$, the performance of the proposed algorithm is far better than the traditional algorithm shown in figure 4 .

\section{CONCLUSIONS}

This paper establishes a food safety production management and quality trace system from the information perspective aiming at food as the research object. The paper detailed introduces the implementation of RFID anticollision algorithm and the collision mechanism in tags identification. Then it proposes a dynamic frame time slot Aloha anti-collision algorithm to fulfill the function of tags anti-collision function in the food safety quality trace system.

\section{ACKNOWLEDGMENT}

2012 Modern agricultural technology system-

Beijing Poultry innovation team Project number:PXM2012014207-000166-00014659-FCG

\section{REFERENCES}

[1] L. Biao, H. Ai qun, and Q. Zhong yuan. Trends and Brief Comments on Anti-collision Techniques in Radio Frequency Identification System [A]. In 6th International Conference on ITS Telecommunications Proceedings[C]. New York: IEEE Communications Society, 2006: 241-245.

[2] Mamatha Nanjundaiah, Vipin Chaudhary. Rovement to the Anticollision Protocol Specification for 900MHz Class 0 Radio Frequency Identification Tag [A], Proceedings of the 19th International Conference on Advanced Information Networking and Applications (AINA' 05)[C]. New York: IEEE Communications Society, 2005, 2: 616-620. 
[3] S. R. Lee, S. D. Joo, and C. W. Lee. An enhanced dynamic framed slotted ALOHA algorithm for RFID tag identification [A]. Second Annual International Conference on Mobile and Ubiquitous Systems Networking and Services[C]. New York: IEEE Computer Society, 2005, 13: 166-172.

[4] J.Eom and T.J.Lee. Frame-slotted Aloha with estimation by pilot frame and identification by binary selection for RFID anti-collision
[A]. International Symposium on Communications and Information Technologies[C]. New York: IEEE, 2007, 1027-1031.

[5] Harald Vogt. Efficient Object Identification with Passive RFID Tags [A]. International Conference on Systems, Man and Cybernetics[C]. New York: IEEE, 2002, 3: 98-113.

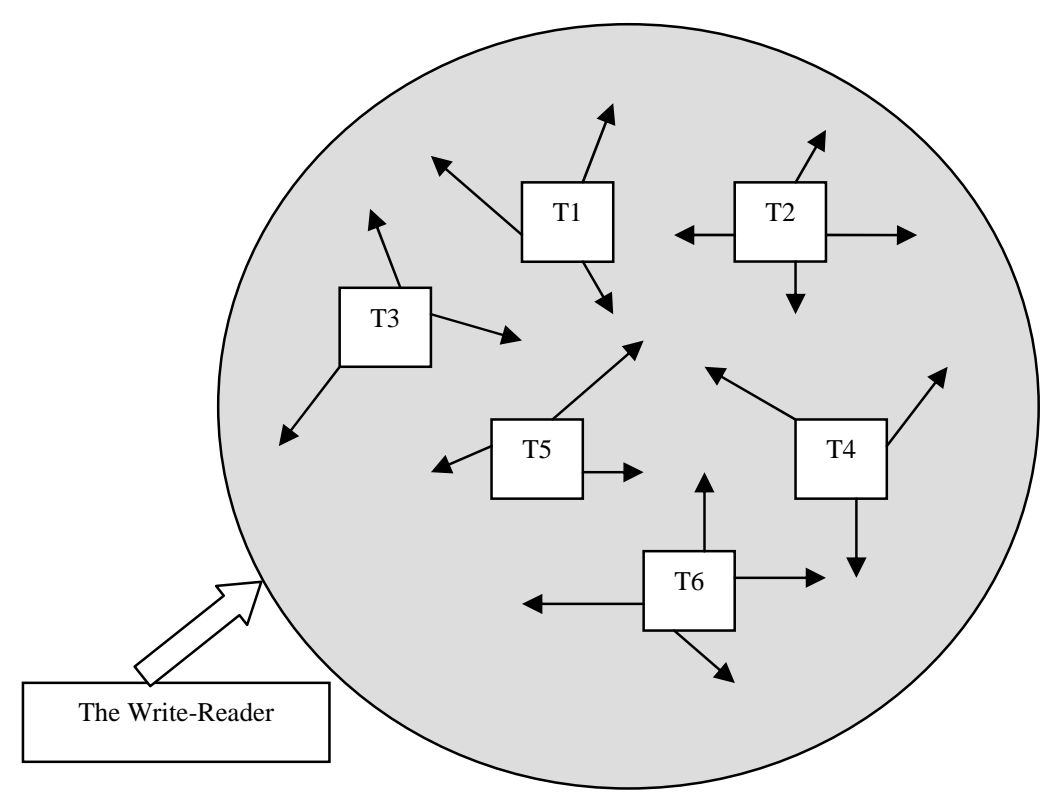

Figure 1. Diagram of tags collision

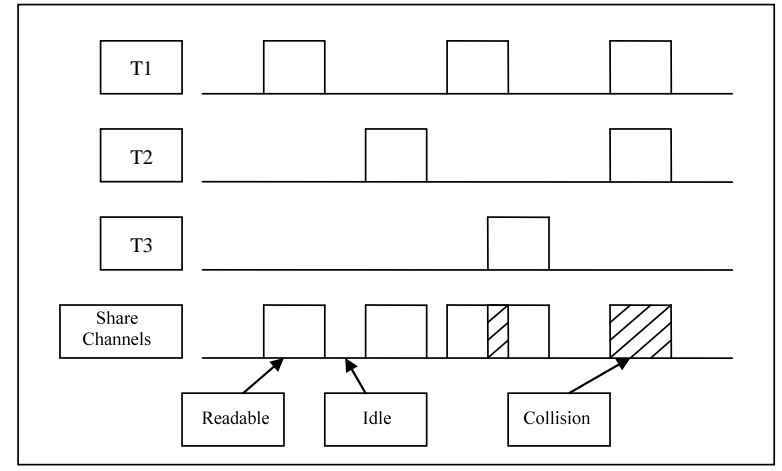

Figure 2. Diagram of pure Aloha algorithm 


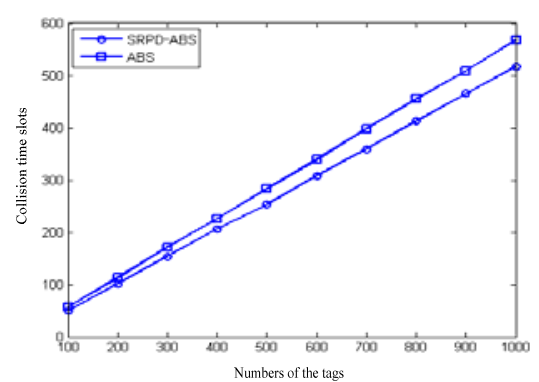

(a) Collision time slots

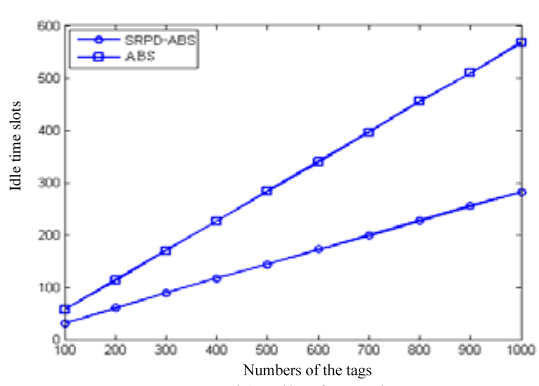

(b) Idle time slots

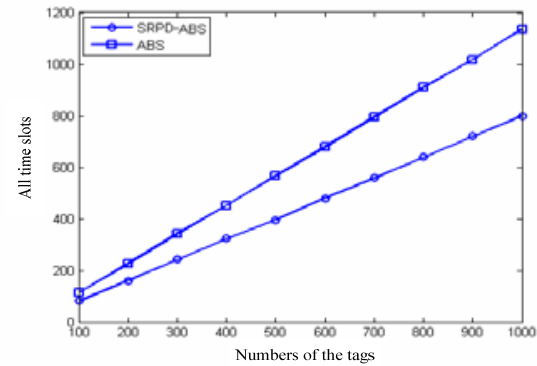

(c) All time slots

Figure 3. Simulation of the proposed algorithm and traditional algorithm when the number of tags is different

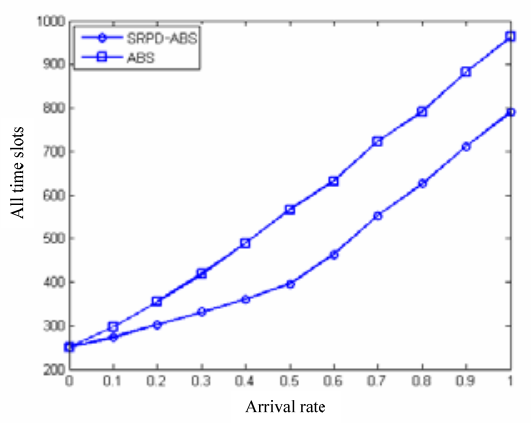

Figure 4. The effect of RA on identification delay when $\mathrm{RL}=0.5$

TABLE I. THE OPERATION OF FRAME TIME SLOT ALOHA ALGORITHM

\begin{tabular}{|l|l|l|c|c|c|c|c|c|c|c|}
\hline \multicolumn{2}{|c|}{} & \multicolumn{3}{c|}{ Time Slot } & \multicolumn{5}{c|}{ Time Slot } \\
\hline Down link & Request & $\mathbf{1}$ & $\mathbf{2}$ & $\mathbf{3}$ & $\mathbf{4}$ & Request & $\mathbf{1}$ & $\mathbf{2}$ & $\mathbf{3}$ & $\mathbf{4}$ \\
\hline Up link & & collision & collision & collision & 0001 & & 1111 & collision & 1100 & collision \\
\hline T1 & & 1011 & & & & & & 1011 & & \\
\hline T2 & & & & 1100 & & & & & 1100 & \\
\hline T3 & & 1111 & & & & & 1111 & & & \\
\hline T4 & & & & & 0001 & & & & & \\
\hline T5 & & & & 1010 & & & & 1010 & & \\
\hline
\end{tabular}

TABLE II. THE OPERATION OF DYNAMIC FRAME TIME SLOT ALOHA ALGORITHM

\begin{tabular}{|c|c|c|c|c|c|c|c|c|c|c|c|}
\hline \multirow[b]{2}{*}{ Down link } & \multirow[b]{2}{*}{ Request } & \multicolumn{3}{|c|}{ Time Slot } & \multirow[b]{2}{*}{ Request } & \multicolumn{6}{|c|}{ Time Slot } \\
\hline & & 1 & 2 & 3 & & 1 & 2 & 3 & 4 & 5 & 6 \\
\hline Up link & & collision & collision & collision & & 1111 & collision & 1100 & collision & 1111 & 0100 \\
\hline T1 & & 1011 & & & & & 1011 & & & & \\
\hline $\mathrm{T} 2$ & & & 1100 & & & & & 1100 & & & \\
\hline T3 & & 1111 & & & & 1111 & & & & 1111 & \\
\hline $\mathrm{T} 4$ & & & & 0001 & & & & & 0001 & & \\
\hline T5 & & & 1010 & & & & 1010 & & & & \\
\hline T6 & & & & 0010 & & & & & 0010 & & \\
\hline T7 & & & & 0100 & & & & & & & 0100 \\
\hline
\end{tabular}

